Thomas Raiser

\title{
Aufgaben der Rechtssoziologie als Zweig der Rechtswissenschaft
}

Antrittsvorlesung

24. Juni 1993

Humboldt-Universität zu Berlin

Fachbereich Rechtswissenschaft 
Mit Fußnoten versehener Text der Antrittsvorlesung an der HumboldtUniversität zu Berlin. Die Form der mündlichen Rede ist beibehalten.

Herausgeberin:

Die Präsidentin der Humboldt-Universität zu Berlin

Prof. Dr. Marlis Dürkop

Copyright: Alle Rechte liegen beim Verfasser.

Redaktion:

Christine Gorek

Forschungsabteilung der Humboldt-Universität

Unter den Linden 6

10099 Berlin

Herstellung:

Linie DREI, Agentur für Satz und Grafik

Wühlischstraße 33

10245 Berlin

\section{Heft 13}

Redaktionsschluß:

2. 8.1993 


\section{Wurzeln der Rechtssoziologie in Berlin}

Die Rechtssoziologie ist nach wie vor das am wenigsten etablierte Fach der Juristenausbildung, und in der Rechtswissenschaft führt sie bis heute ein Außenseiterdasein. Längst nicht alle Universitäten bieten rechtssoziologische Lehrveranstaltungen an. Im Staatsexamen spielt das Fach praktisch keine Rolle. Rechtssoziologische Forschung wird in der Bundesrepublik nur an wenigen Stellen und mit ganz unzulänglichen personellen und finanziellen Mitteln betrieben. Nicht wenige Juristen scheinen angesichts der Disziplin weiterhin ein gewisses Unbehagen zu verspüren. Dieses mag sich zum Teil aus den anderen Fragestellungen und den nicht geläufigen Methoden der empirischen Sozialforschung erklären. Es spiegelt aber doch wohl auch noch immer ein gewisses Mißtrauen gegenüber dem aufklärerischen, kritischen und innovativen Impuls des Fachs wider. Die dogmatische Jurisprudenz hat prinzipiell vom positiven Recht und den darin verfestigten Herrschaftsstrukturen auszugehen. Diese zu hinterfragen, ist gewöhnlich nicht ihr Ziel. So ist es verständlich, daß sie sich von einem Fach herausgefordert fühlen kann, das eine solche Schranke seiner Bestimmung nicht anerkennt.

Warum erwähne ich diese Hintergründe? Zunächst weil sie mir Gelegenheit geben, im Gegensatz dazu die leuchtende Spur gerade der Berliner Juristischen Fakultät in der Rechtssoziologie zu rühmen. Georg Simmel, einer der Väter der deutschen Soziologie, dem wir auch wichtige rechtssoziologische Erkenntnisse verdanken ${ }^{1}$, hat den größten Teil seines Lebenswerkes in Berlin geschrieben. Von den Juristen der Jahrhundertwende ist als erster Franz von Liszt zu nennen, der unter dem Einfluß Rudolf von Jherings gegen viele Widerstände die soziologische Betrachtungsweise in die Kriminalwissenschaften eingeführt hat. Jhering 
selbst war ein halbes Jahrhundert vorher in Berlin habilitiert worden, ist dann allerdings nicht mehr hierher zurückgekehrt. Ein Schüler von Liszt war Hermann Kantorowicz, der Verfasser der berühmten, 1906 unter dem Decknamen Gnaeus Flavius veröffentlichten Streitschrift „Der Kampf um die Rechtswissenschaft“. Dieses Manifest der sogenannten Freirechtslehre hat bekanntlich der steril gewordenen Begriffsjurisprudenz des 19. Jahrhunderts den Todesstoß versetzt. Sein Nährboden war ein Kreis junger Juristen hier in Berlin, zu dem übrigens auch Gustav Radbruch gehörte. Es ist trotz der offenkundigen Übertreibungen eines jugendlichen Bilderstürmers eine der bedeutendsten, meistgelesenen und wirkungsvollsten juristischen Schriften dieses Jahrhunderts geworden ${ }^{2}$.

Methodisch weniger revolutionär, dafür auf andere Weise wegweisend, waren zur selben Zeit die Berliner Initiativen zur Rechtstatsachenforschung. Gleichfalls 1906 organisierte Martin Wolff, der Autor des berühmten Sachenrechtslehrbuches, die erste große Fragebogenaktion, um festzustellen, welche ,tatsächliche Bedeutung den einzelnen Rechtsinstituten des BGB im Leben des deutschen Volkes“ zukomme. Er wollte „ein klares Bild der Sitten des deutschen Rechtslebens“" gewinnen" ${ }^{3} 1914$ veröffentlichte Arthur Nußbaum, Berliner Rechtsanwalt und später bis zu seiner Vertreibung 1933 Fakultätsmitglied, seine große Programmschrift „Die Rechtstatsachenforschung - ihre Bedeutung für Wissenschaft und Unterricht" "4. Ihr folgte eine lange Serie von rechtstatsächlichen Untersuchungen Nußbaums und seiner Schüler.

Rühmenswert ist nicht zuletzt die Hinwendung der Fakultät zur empirischen rechtssoziologischen Forschung seit Beginn der achtziger Jahre. Daraus ist eine eindrucksvolle Liste von Untersuchungen zum Strafrecht, allgemeinen Zivilrecht, Familienrecht, Arbeitsrecht und zur Rechtspflege hervorgegangen. Sie sind überwiegend in die Implementations- und Effektivitätsforschung einzuordnen 5 . Schon vor der Wende gab es auch Initiativen zu einem stärkeren Ausbau und einer institutionellen Absicherung der Rechtssoziologie an der Fakultät ${ }^{6}$. An alle diese Vorbilder gilt es heute anzuknüpfen. 
Nun habe ich aber angekündigt, über Aufgaben der Rechtssoziologie zu sprechen, also den Blick in die Zukunft zu richten. Was wird von der Rechtssoziologie heute erwartet? Was kann sie leisten? Was hat sie zu den juristischen Methoden, zum Erkenntnisfortschritt, zur Verbesserung von Recht und Rechtspflege beizutragen?

Ich werde versuchen, dies mit Hilfe von zwei Beispielen herauszuarbeiten, von denen das erste der empirischen Feldforschung entstammt, das zweite der sozialwissenschaftlichen Theorie. Zunächst beziehe ich mich auf das Forschungsprojekt zur Wirkung der Rechtsschutzversicherungen auf das Prozeßverhalten, das mich selbst mehrere Jahre beschäftigt hat und nunmehr vor seinem Abschluß steht. Anschließend gehe ich auf die Geltungsgründe des positiven Rechts ein und setze mich dabei mit den Lehren von Niklas Luhmann und Jürgen Habermas auseinander.

\section{Doppelnatur der Rechtswissenschaft}

Zuvor sind noch einige Worte zum methodischen Ausgangspunkt nötig. Die Formulierung des Themas setzt voraus, daß die Rechtssoziologie ein Zweig der Rechtswissenschaft ist. Das versteht sich keineswegs von selbst. Schon die Bezeichnung legt es nahe, sie eher der Soziologie zuzuordnen. Solange in der Tradition des Neukantianismus zwischen den Reichen des Seins und des Sollens ein unüberwindlicher Graben gesehen wurde, führte auch keine Brücke von der als Seinswissenschaft verstandenen Soziologie zur normativen Jurisprudenz. Noch heute betont kein Geringerer als Niklas Luhmann mit Nachdruck die Distanz zwischen den Disziplinen. Er schreibt, die Rechtssoziologie könne das Recht nur von außen betrachten, aber schlechterdings nichts zur Entscheidung offener Rechtsfragen beitragen?

Ich sehe das anders und begründe es auf dreifache Weise. Zunächst abstrakt: Wenn das Recht zum Gegenstand der Wissenschaft gemacht wird, wäre es unwissenschaftlich, eine Disziplin auszuschließen, die sich mit eben diesem Thema beschäftigt. 
Wichtiger ist mir zweitens die Beobachtung, daß schlechterdings keiner der Versuche gelungen ist, die Rechtssoziologie nach Gegenstand oder Methode von den anderen Zweigen der Rechtswissenschaft, namentlich von der Rechtsphilosophie oder der Rechtsdogmatik, zu trennen. Man kann zum Beispiel nicht sagen, die Rechtsdogmatik arbeite als Geistes- und Buchwissenschaft stets mit den Mitteln der hermeneutischen Interpretation, die auf das Verstehen von Sinn gerichtet sind, während die Rechtssoziologie auf die Erkenntnis der Rechtswirklichkeit zielt und sich dazu der empirischen Methoden der Beobachtung und des Versuchs bedient. Denn auch die Rechtsdogmatik verschließt ihren Blick keineswegs vor der Rechtswirklichkeit, und die Rechtssoziologie kann andererseits auf die verstehende Analyse von Texten nicht verzichten. Wo eine strikte Trennung der Fächer versucht wird, geht dies regelmäßig auf Kosten einer methodischen Verengung des Blickfelds, die von anderen nicht geteilt wird. In Wahrheit finden wir überall einen Methodenpluralismus und -synkretismus, der die Grenzen verwischt.

Bei solchen Überlegungen gelangen wir drittens letztlich zu der erkenntnis- und wissenschaftstheoretischen Frage nach dem Ziel der Rechtswissenschaft. Natürlich lautet die Antwort: Die Erforschung des Rechts. Dieses selbst weist aber eine doppelte Natur auf. Es ist zugleich Sinnträger und Handlungsmuster ${ }^{8}$. Es will erkannt und verwirklicht werden. Deshalb darf auch die Rechtswissenschaft sich nicht auf eine dieser beiden Komponenten beschränken. Im Gegenteil hat sie stets die beiden Kantischen Fragen der reinen und der praktischen Vernunft zu stellen: „Was können wir wissen?" und „Was sollen wir tun?“. Sie hat sich mit anderen Worten zugleich in den Dienst der Erkenntnis und der Verbesserung des Rechts zu stellen. Ihre Perspektive ist sowohl die des Beobachters wie die des Handelnden und Entscheidenden. Oder - um noch einmal im Anschluß an Kant Hermann Kantorowicz zu zitieren: „Dogmatik ohne Soziologie ist leer, Soziologie ohne Dogmatik blind“" . In dieser Sicht ist die Rechtssoziologie in dem Maß ein Zweig der Rechtswissenschaft, in dem sie etwas zur Erkenntnis und zur Verbesserung des Rechts beiträgt. 


\section{Leistungen der rechtssoziologischen Empirie}

Wenden wir uns nach diesen grundsätzlichen Überlegungen der empirischen Rechtssoziologie $\mathrm{zu}^{10}$. Die Rechtsschutzversicherung ist bekanntlich eine private Versicherung, die für den Fall bestimmter Rechtsstreitigkeiten die Anwalts- und Gerichtskosten übernimmt. Sie hat in der Bundesrepublik seit den 60er Jahren einen gewaltigen Aufschwung genommen. Ihr Prämienaufkommen verdoppelte sich allein in den 10 Jahren zwischen 1980 und 1990 und belief sich 1990 auf etwa 3,2 Milliarden DM. Über ihre Auswirkungen auf das Verhalten der Versicherten und der Rechtsanwälte sowie auf die Belastung der Gerichte herrscht seit langem heftiger Streit. Die Alltagsmeinung, die auch von den Richtern geteilt wird, sagt, Rechtsschutzversicherte prozessieren häufiger und hartnäckiger als Nichtversicherte, und sie nehmen die Gerichte in zahlreichen unbegründeten Fällen in Anspruch. Michael Adams hat diesen Standpunkt wahrscheinlichkeitstheoretisch untermauert und gelangt zu dem Ergebnis, daß die Rechtsschutzversicherungen eine geradezu sozialschädliche Prozeßlawine auslösen und daher verboten, mindestens stark beschränkt gehören ${ }^{11}$.

Auf der anderen Seite betonen namentlich die Versicherer und die Rechtsanwälte die soziale Komponente dieses Versicherungszweiges, der zahlreichen Personen ermögliche, ihr gutes Recht auch durchzusetzen, worauf sie anderenfalls wegen der hohen Kosten verzichten müßten. Sie bezweifeln eine prozeßsteigernde Wirkung mit dem Argument, die Anwälte würden ihren Mandanten aussichtslose Klagen auch dann ausreden, wenn diese gegen die Kosten versichert sind ${ }^{12}$. Wissenschaftlich können sie sich auf eine empirische Untersuchung von Blankenburg und Fiedler stuitzen, die zu dem Ergebnis kamen, außer bei Streitigkeiten rund um das Auto sei eine Vermehrung der Gerichtsverfahren nicht festzustellen ${ }^{13}$.

Angesichts der Unklarheiten über die tatsächliche Lage ließen sich jahrelang in aller Öffentlichkeit ausgetragene Kontroversen über die Rechtsschutzversicherungen nicht klären. Politische 
Forderungen nach einem gesetzlichen Eingriff konnten sich weder durchsetzen noch gelang es, sie abzublocken. Dies war der Anlaß für das Bundesministerium der Justiz, zu dem Thema einen neuen Forschungsauftrag zu vergeben.

Wir haben etwa 750 Rechtsanwälte über ihre Erfahrungen mit den Rechtsschutzversicherungen befragt und über 5.200 Anwaltsakten in den Bereichen Zivilrecht, Arbeitsrecht, Verkehrsstrafrecht und Verkehrsordnungswidrigkeiten ausgewertet. Das Ziel war, statistische Unterschiede zwischen Versicherten und Nichtversicherten hinsichtlich Häufigkeit und Hartnäckigkeit der gerichtlichen Rechtsverfolgung herauszufinden. Außerdem wollten wir wissen, ob Rechtsschutzversicherte weniger erfolgreich sind als Nichtversicherte.

Ich verzichte hier auf alle Einzelheiten und berichte gleich über die Ergebnisse. Sie vermitteln ungeachtet deutlicher Unterschiede im einzelnen ein Gesamtbild, wonach Versicherte in der Tat häufiger und hartnäckiger prozessieren als Nichtversicherte. Aber die Unterschiede liegen regelmäßig bei nur 5\% bis $10 \%$. Nur in einzelnen Bereichen, namentlich bei Verkehrsordnungswidrigkeiten und bei Schadensersatzklagen nach Verkehrsunfällen, gehen sie über $10 \%$ hinaus. Die Versicherten sind auch weniger erfolgreich, aber in noch geringerem Maße. Vielmehr hat ein Teil der zusätzlich eingelegten Klagen ganz oder doch wenigstens teilweise Erfolg. Im Zivilrecht und im Arbeitsrecht fanden sich auch Fallgruppen, in denen Versicherte trotz der höheren Prozeßquote gleich erfolgreich sind wie Nichtversicherte.

Was bedeuten nun diese Befunde für die Erkenntnis und für die Verbesserung des Rechts? In der abschließenden Beratung der Ergebnisse mit den Vertretern der Rechtsanwaltschaft, der Versicherungswirtschaft, der Richter und der Justizverwaltungen im Bundesjustizministerium waren - siehe da - alle zufrieden und deuteten ihre Bereitschaft an, die öffentlichen Kontroversen um den Gegenstand nunmehr zu beenden. Es gab keine nachhaltigen Einwände gegen unseren Befund, daß gravierende Mißstände nicht aufgetreten seien und der zusätzliche Geschäftsanfall der 
Gerichte infolge ungerechtfertigter, durch die Rechtsschutzversicherung veranlaßter Klagen gering sei. So ließ sich auch ein Konsens zu unserer Schlußfolgerung herstellen, gesetzliche Eingriffe in das System der Rechtsschutzversicherungen und Änderungen des Versicherungsrechts zu diesem Zweck seien nicht dringlich. Wenn man wegen der zu hohen Geschäftslast der Gerichte Beschränkungen des Rechtsschutzes für notwendig hält, sollten andere Mittel vorgezogen werden, die gegenüber allen wirken und Rechtsschutzversicherte nicht einseitig benachteiligen.

Ziehen wir die Konsequenzen für unser Thema: Das ist es, was die empirische Rechtssoziologie als Zweig der Rechtswissenschaft leisten kann: Erarbeitung solider Kenntnis der Rechtstatsachen; Bereinigung von Vorurteilen und Alltagstheorien, die auf einer selektiven Wahrnehmung der Rechtswirklichkeit beruhen, und der darauf zurückgehenden politischen Kontroversen und Blockaden. Formulierung sachlich begründeter und somit aussichtsreicher Vorschläge zur Verbesserung des Rechts.

\section{Das Problem des Rechtspositivismus}

Ich komme zur Theorie und damit zu den Geltungsgründen des Rechts. Das soziologische Denken war seit seinem Gründer Auguste Comte vom wissenschaftlichen Positivismus gekennzeichnet, das heißt von dem Bemühen, die soziale Realität ohne Rückgriff auf religiöse und metaphysische Denkmuster zu erforschen. In der Rechtssoziologie fand dieser Ansatz im Rechtspositivismus seinen Ausdruck. Dieser begreift als Recht das gesetzte und tatsächlich praktizierte Recht, dessen Geltung sich allein auf die Praktizierungen von seiten der Rechtsgenossen oder die rechtssetzende und -durchsetzende Gewalt des Staates stützt, aber keiner Rechtfertigung unter dem Gesichtspunkt eines übergeordneten Kriteriums der Gerechtigkeit bedarf. Alle großen Rechtssoziologen der ersten Hälfte des Jahrhunderts und übrigens auch die meisten Rechtsphilosophen stehen in dieser Tradition. Ich nenne nur Eugen Ehrlich, Max Weber, Hans Kelsen, Theodor 
Geiger. Bei ihnen allen bleibt die Frage nach der sozialen Gerechtigkeit daher auf eigentümliche Weise ausgespart oder doch unterbelichtet.

In unserer Zeit wird der soziologische Rechtspositivismus am nachdrücklichsten von Niklas Luhmann vertreten. Luhmann behauptet, in den hochdifferenzierten modernen Gesellschaften sei ein auf übergeordnete Prinzipien der Gerechtigkeit gegründetes Recht nicht mehr möglich, denn ein solches setze die Vorstellung einer unabänderlichen Geltung voraus und wäre deshalb nicht imstande, sich an die sozialen Veränderungen anzupassen und neu auftretende Konflikte zu bewältigen. Nur ein Recht, das jederzeit geändert werden kann und dessen Änderung von vornherein vorgesehen ist, das inhaltlich aber beliebig bleibt, könne die ihm gestellte doppelte Aufgabe lösen, ein soziales System einerseits zu stabilisieren, andererseits gegenüber wechselnden Umweltanforderungen anpassungsfähig zu halten ${ }^{14}$.

Der Vorteil des positiven Rechts liege also in einer außerordentlichen Steigerung seiner Komplexität, die mit der pluralistischen Gesellschaft Schritt halten kann. Diese Eigenschaft ermögliche, vergleichbare Sachverhalte unterschiedlich zu regeln, weil das Bedürfnis nach innerer Widerspruchsfreiheit des ganzen Rechtssystems angesichts der Segmentierung der Anwendungsbereiche geringer werde. Eben dadurch werde das Recht auch zum brauchbaren Instrument für die Steuerung gesellschaftlicher Prozesse, das heißt für die planmäßige Sozialgestaltung. All dies verlange aber die strikte Trennung von Recht und Wahrheit sowie Recht und Moral ${ }^{15}$. Die mit der gewollten Beliebigkeit von Rechtsänderungen verknüpfte Gefahr mangelnder Akzeptanz von seiten der Normadressaten wird nach Luhmann kompensiert durch die Schutzvorkehrungen des Rechtsstaats und durch die Einrichtung förmlicher Verfahren der Rechtsänderung, die ihrerseits rechtlich geregelt sind ${ }^{16}$.

In der Theorie der Autopoiese sozialer Systeme formuliert Luhmann den Sachverhalt wie folgt ${ }^{17}$ :

„Wenn man .... die Positivierung des Rechts als ein Korrelat der 
Ausdifferenzierung des Rechtssystems und seiner autopoietischen Autonomie auffaßt, ...ist Rechtsgeltung gar nicht anders möglich als ,positiv“, das heißt: durch das Recht selbst gesetzt. Das Recht kann regeln, wie es sich selbst reproduziert, das heißt, wie man mit Recht von Recht zu Recht kommt; aber nur das Recht kann dies regeln. Es gibt keine externen Instanzen oder Autoritäten, die Recht in das Recht eingeben könnten. Das Recht wird zirkulär konstituiert, und ein Beobachter, der dies als Einheit beschreiben will, muß deshalb zu einer tautologischen Formulierung greifen. Alle Beschränkungen sind Selbstbeschränkungen, alle Umweltorientierungen müssen im System durch das System gehandhabt werden. Das Recht gilt demnach allein deshalb, weil entschieden worden ist, daß es gilt.“

Immerhin wehrt sich Luhmann gegen den Vorwurf eines rein willkürlichen, prinzipienlosen Dezisionismus. Er führt dagegen an, Recht, das sich nicht bewährt, könne eben jederzeit verbessert werden. Nicht geändertes Recht habe deshalb die Vermutung für sich, gutes Recht zu sein. Auch könne nicht alles Recht auf einmal geändert werden. Eine Richtigkeitsgewähr liege in der Notwendigkeit, jede Rechtsänderung auf das fortgeltende Recht abzustimmen. Weiteren Schutz gegen Willkür biete die Differenziertheit der Rechtsmaterien, die dazu führe, daß jede einzelne Rechtsänderung immer nur begrenzte Wirkungen entfalten $\mathrm{kann}^{18}$.

Luhmanns Positivismus entspricht, wie bereits ausgeführt, der rechtssoziologischen Tradition. $\mathrm{Zu}$ seinen Gunsten spricht unsere Erfahrung, daß die Rechtsvorschriften in der Tat sozusagen täglich massenhaft geändert werden. Dabei geht es in der Regel um Steuerung der Gesellschaft und Sozialgestaltung nach politischen Zielvorgaben und Kompromissen. Auf moralische Prinzipien wird selten Bezug genommen. Luhmann ist auch zuzustimmen, wenn er sagt, ohne ,strukturelle Variabilität des Rechts“"wäre die Balance zwischen Stabilität und Anpassungsfähigkeit der Gesellschaft kaum zu lösen. Richtig ist weiter, daß die Menschen gewöhnlich bereit sind, Rechtsvorschriften hinzunehmen, wenn sie nur ordnungsgemäß erlassen wurden, und daß vor allem die staat- 
liche Bürokratie und die Gerichte sich unter diesen Voraussetzungen regelmäßig daran orientieren und danach entscheiden. Der Glaube an die Legitimität der legalen Ordnung ist - um Max Webers Terminologie aufzugreifen - auch in der Gegenwart die wohl wichtigste Stütze der sozialen Integration. Aus diesen Gründen wird auch die Rechtssoziologie den Positivismus nicht einfach aufgeben können.

Die Frage ist aber, ob er so verabsolutiert werden darf, wie dies bei Luhmann geschieht. Dagegen sprechen wichtige Gründe. Ich nenne hier zunächst nur unsere historischen Erfahrungen. Der grenzenlose Machtmißbrauch des Hitler-Regimes, das sich zur Durchsetzung seiner Herrschaft nicht zuletzt des Mittels von Rechtsvorschriften bediente, hat den naiven Glauben an die uneingeschränkte Legitimität des positiven Rechts unmöglich gemacht. Berühmt geworden ist die vorsichtige Korrektur, die Gustav Radbruch 1946 formuliert hat, und die ins Gedächtnis zu rufen sich immer wieder lohnt:

„Der Konflikt zwischen der Gerechtigkeit und der Rechtssicherheit dürfte dahin zu lösen sein, daß das positive, durch Satzung und Macht gesicherte Recht auch dann den Vorrang hat, wenn es inhaltlich ungerecht und unzweckmäßig ist, es sei dann, daß der Widerspruch des positiven Gesetzes zur Gerechtigkeit ein so unerträgliches Maß erreicht, daß das Gesetz als ,unrichtiges Recht“ der Gerechtigkeit zu weichen hat ${ }^{19 \%}$.

Ähnliche Probleme beschäftigen uns heute, wenn wir fragen müssen, ob nicht manche Gesetze der DDR Unrecht waren, ob nicht etwa der Schießbefehl an der Mauer gegen fundamentale Prinzipien der Gerechtigkeit verstieß und daher nicht befolgt werden durfte. Umgekehrt artikuliert sich die Skepsis gegenüber dem positiven Recht der Bundesrepublik in dem zum geflügelten Wort gewordenen Ausspruch von Frau Bohley: ,Wir haben Gerechtigkeit erwartet und statt dessen den Rechtsstaat bekommen.“

Sehen wir uns demnach genötigt, nun doch zwischen gerechten und ungerechten Gesetzen zu unterscheiden, so fangen die Schwierigkeiten allerdings erst an. Sie liegen hauptsächlich in der 
Unsicherheit darüber, welche Regelungen eigentlich gerecht sind, denn in der pluralistischen Gesellschaft der Gegenwart finden sich dazu nicht ohne weiteres einheitliche Wertvorstellungen und ein allgemein anerkanntes Modell einer gerechten Sozialordnung. Auch verbietet es unser Leitbild vom sittlich autonomen Menschen dem Gesetzgeber, sich auf ein solches Modell festzulegen. Jeder Materialisierung des Rechts wohnt eine Tendenz zur Bevormundung des Menschen inne, bis hin zur Willkür und zum Autoritarismus. Eine Rechtsordnung, die ihren Geltungsanspruch auf formale Legalität gründet, kann sich in dieser Sicht auch als Garant einer liberalen Ordnung verstehen.

Den Positivismus zu überwinden und zugleich die Gefahren einer autoritären Materialisierung des Rechts zu vermeiden, ist das Ziel des neuen Buchs von Jürgen Habermas „Faktizität und Geltung "20. Positives Recht ist auch für Habermas in den differenzierten und pluralistischen Gesellschaften der Gegenwart das wesentliche Medium sozialer Integration ${ }^{21}$. Um diese Funktion erfüllen zu können, genügen aber Setzung und Durchsetzung von Normen beliebigen Inhalts nicht. Das Recht muß seinerseits legitim sein ${ }^{22}$. Wodurch erlangt es Legitimität?

Im wesentlichen sind es vier Elemente, die Habermas' Gedanken stützen. Lassen Sie mich diese in aller Kürze skizzieren:

1. Rechtssetzung geschieht in einem rationalen, selbst rechtlich geregelten Verfahren, an dem die Staatsbürger als politisch autonome Rechtssubjekte teilnehmen. Legitimität gewinnt das Recht in diesem Verfahren ,,durch die sozialintegrative Kraft des übereinstimmenden und vereinigten Willens aller freien und gleichen Staatsbürger" ${ }^{\text {“23 }}$. Hier beruft sich Habermas auf die Lehren vom $l i$ beralen und demokratischen Rechtsstaat, als deren Väter er ausdrücklich Rousseau und Kant zitiert.

2. Die Kommunikation zwischen den Bürgern geschieht durch Diskurs. Der Begriff des Diskurses ist ein Kernbegriff in Habermas' Theorie des kommunikativen Handelns. Diskurse vollziehen sich in Gestalt der Sprache, die eine wechselseitige Verständigung über Geltungsansprüche und damit über gemeinsame Handlungsorientierungen und soziale Normen ermöglicht ${ }^{24}$. Es gibt viele Arten von Diskursen: meinungsbildende und entschei- 
dungsvorbereitende ${ }^{25}$; ethische, moralische, pragmatische, Verhandlungsdiskurse und eben rechtliche Diskurse ${ }^{26}$; letztere zielen darauf ab, die Konsistenz von Rechtsentscheidungen sicherzustellen.

3. Die Diskurse sind vernünftig, weil sie von vernünftigen und freien Individuen geführt werden und übereinstimmende Muster des Denkens und Handelns herbeiführen. Die kommunikative Vernunft schafft Gesetze, ,unter denen die Willkür des einen mit der Willkür des anderen nach einem allgemeinen Gesetz der Freiheit vereinigt werden kann"“27. Ist diese Bedingung erfüllt, ist ein Gesetz legitim, denn es ist ,,beides zugleich garantiert: sowohl die Legalität des Verhaltens im Sinn einer durchschnittlichen Normbefolgung, die erforderlichenfalls durch Sanktionen erzwungen wird, als auch die Legitimität der Regel selbst, die eine Befolgung der Norm aus Achtung vor dem Gesetz jederzeit möglich macht ${ }^{\text {" } 28}$. Mit diesen Ausführungen greift Habermas, wie Sie sicher sogleich herausgehört haben, zum zweiten Mal auch in der Formulierung auf die formale Rechtslehre Kants zurück.

4. Im Gegensatz zu den rechtlichen Diskursen fragen moralische Diskurse nach Regeln, die von der ganzen Menschheit akzeptiert werden können und deshalb keiner demokratischen Setzung bedürfen ${ }^{29}$. Dazu gehört auch der Diskurs über die Gerechtigkeit. Moralische Diskurse verbleiben aber in der pluralistischen Gesellschaft im Bereich des kulturellen Wissens und symbolischer Geltung. Moralische Normen erlangen nicht die institutionelle, durch Zwang gesicherte Verbindlichkeit des Rechts. Moral und Recht stehen auch nicht in einem Verhältnis der Über- und Unterordnung, sondern autonom nebeneinander. Sie erfüllen unterschiedliche Funktionen und ergänzen sich wechselseitig ${ }^{30}$. Immerhin bleibt die Moral mit dem Rechtssystem verknüpft und kann auf alle seine Bereiche ,ausstrahlen “ ${ }^{\text {“31 }}$. Habermas rechnet sogar damit, daß Gesetzgebung und Gesetze einem ,sensiblen moralischen Test ausgesetzt" werden. Er verlangt deshalb eine vorbehaltlose Öffnung des Normsetzungsverfahrens für den sich in der öffentlichen Meinung vollziehenden moralischen Diskurs $^{32}$. 
Kehren wir nach dieser wiederum sehr gerafften Darstellung zu der Frage zurück: Wie bewältigt Habermas das Problem des Rechtspositivismus? Soviel ist klar: Recht beliebigen Inhalts, das allein durch seine Gesetztheit legitimiert ist, kann es bei ihm nicht geben. Aber er stellt auch keinen Bezug zu inhaltlich gehaltvollen Maximen einer materiellen Gerechtigkeit her, sondern siedelt diese in einem vom Recht getrennten, autonomen Diskurs der Moral an. Die Legitimitätsgrundlage des positiven Rechts ist demgegenüber allein der rechtliche Diskurs und das rationale und demokratische Gesetzgebungsverfahren. Beide gewährleisten, daß die Autonomie des Individuums und gleichzeitig die Akzeptabilität der gesetzten Normen in größtmöglichem Ausmaß gesichert wird.

\section{Rechtspositivismus und Rechtswissenschaft}

Für den Juristen ist auch dieses zu wenig, wie ich zum Schluß in drei Schritten zeigen will ${ }^{33}$ :

1. Das Grundgesetz, das den Maßstab für alle Rechtsbildung in der Bundesrepublik festlegt, geht unzweifelhaft von einem nicht nur prozeduralen, sondern inhaltlich gehaltvollen Gerechtigkeitsverständnis aus. Dies kommt zum Beispiel in der Bindung aller staatlichen Gewalt an den obersten Wert der Menschenwürde in Art. 1 GG, in der Bezugnahme auf Gesetz und Recht in Art. 20 Abs. 3 GG, schwächer in der Sozialstaatsklausel (Art. 20 Abs. 1, 28 Abs. 1 GG), weiter aber auch zum Beispiel im besonderen Schutz von Ehe und Familie (Art. 6 GG) oder im Verbot der Todesstrafe (Art. 102 GG) zum Ausdruck. Demgemäß hat das Bundesverfassungsgericht unter Zustimmung des größten Teils der Literatur die Grundrechte von Anfang an nicht allein als gegen den Staat gerichtete subjektive Freiheitsrechte verstanden, sondern als Ausdruck einer objektiven Wertordnung, die alle Rechtsverhältnisse ergreift ${ }^{34}$. Auch das Strafgesetzbuch und das Bürgerliche Gesetzbuch beziehen sich übrigens in den Verweisen auf die guten Sitten auf eine über dem positiven Recht stehende Wertordnung als Bestandteil des geltenden Rechts. 
2. Die lediglich prozedurale Legitimation der Rechtssetzung hilft in keinem Fall, die großen Probleme einer gerechten Ordnung unserer sozialen Lebensverhältnisse zu lösen. Schauen wir uns um: Schwangerschaftsabbruch, Aufnahme von Asylanten und Ausländern, Einkommens-, Eigentums- und Vermögensverteilung und -umverteilung, soziale Sicherheit und Gewährleistung des Existenzminimums, Steuergerechtigkeit, Verbraucherschutz, Garantie gleicher Bildungschancen: überall sind materielle Gerechtigkeitskriterien gefragt, ohne die die Politik richtungslos, wenn nicht willkürlich bleibt und ohne deren Überzeugungskraft die Akzeptanz der im politischen Prozeß gefundenen Lösungen schwerlich erwartet werden kann.

3. Auf der anderen Seite erscheint die Skepsis, in der modernen, hochdifferenzierten und pluralistischen Gesellschaft lasse sich ein Konsens über inhaltliche Gerechtigkeitsmaßstäbe nicht finden, in ihrer uneingeschränkten und unkritischen Verallgemeinerung überzogen. In der alten Bundesrepublik herrschte ein hohes $\mathrm{Maß}$ an Zustimmung zu der im Grundgesetz geformten Werteordnung und zum Gerechtigkeitswert der sozialen Strukturen, die auf dieser Grundlage entstanden sind. Auch wenn ein solcher Grundkonsens in den neuen Bundesländern bisher prekär geblieben ist, halte ich ihn nicht für zerbrochen. Auf die solchen Konsens begründenden Gerechtigkeitsvorstellungen müssen Politiker bauen, wenn sie überzeugendes und daher wirksames positives Recht setzen wollen. Auch die Rechtssoziologie wird daher ihrem herkömmlichen Positivismus einen Bezug zu den materiellen Gerechtigkeitswerten hinzufügen müssen, welche die Integration der Gesellschaft tragen. Neuere Arbeiten deuten darauf hin, daß sie diese Dimension ihrer Aufgabe nunmehr auch wahrnimmt ${ }^{35}$.

Damit bin ich am Ende und fasse das Resultat unserer Überlegungen nur noch in zwei Thesen zusammen:

1. Die Rechtssoziologie ist in dem Maße ein Zweig der Rechtswissenschaft, in dem sie sich zur Aufgabe macht, nicht nur zur Erkenntnis, sondern auch zur Verbesserung des Rechts beizutragen.

2. Gelingt ihr dies, ist sie als Zweig der Rechtswissenschaft un- 
verzichtbar, denn die Leistungen der sozialwissenschaftlichen Empirie und der theoretischen Gesellschaftsanalyse werden im Rechtsbildungsproze $ß$ nicht weniger benötigt als dogmatische Jurisprudenz. 


\section{Anmerkungen}

1 Vgl. nur das Kapitel „Der Streit“" in seiner Soziologie von 1908.

2 Kantorowicz hat die Übertreibungen später selbst zugestanden und zurückgenommen. Vgl. dazu meine Abhandlung „Hermann Kantorowicz“" in: Lutter/Stiefel (Hrsg.), Einfluß deutschsprachiger juristischer Emigranten auf die Rechtsentwicklung in den USA und in Deutschland (im Erscheinen).

3 Juristische Wochenschrift 1906, S. 15. Die Ergebnisse wurden veröffentlicht von Sengall im Archiv für Bürgerliches Recht 32 (1908), S. 410 ff.

4 wieder abgedruckt in Rehbinder (Hrsg.), Die Rechtstatsachenforschung, 1968.

5 Darstellung und Analyse im einzelnen bei Kessel, Rechtssoziologie in der Deutschen Demokratischen Republik, Diss. Berlin (FU) 1992.

6 Vgl. Will, Betrifft: Forschungsprofilierung Rechtssoziologie, Zeitschrift für Rechtssoziologie 1990, S. 2; Niederländer, Rechtssoziologie - an DDR-Universitäten ein neues Lehr- und Forschungsgebiet, Zeitschrift für Rechtssoziologie 1990, S. 10.

7 Luhmann, Die soziologische Beobachtung des Rechts, 1986, S.19 ff.

$8 \mathrm{Vgl}$. Habermas, Faktizität und Geltung, S. 146.

9 Kantorowicz, Rechtswissenschaft und Soziologie, 1911, Neudruck 1962, S. 139.

10 Vgl. zum folgenden Jagodzinski/Raiser/Riehl, Rechtsschutzversicherung und Rechtsverfolgung. Eine empirische Untersuchung. Forschungsbericht, erscheint Anfang 1994; Vorwegbericht in: Jagodzinski/Raiser/Riehl, Auswirkungen der Rechtsschutzversicherung auf die Rechtspflege, Zeitschrift für Rechtssoziologie 1991, S. 287 ff.

11 Adams, Ökonomische Analyse des Zivilprozesses, 1981, S. 112 ff; ders., Der Zivilprozeß als Folge strategischen Verhaltens, Zeitschrift für Rechtssoziologie 1986, S. 212 ff; Adams/Blankenburg, Der Einfluß der Rechtsschutzversicherungen auf den Geschäftsanfall der Gerichte. Ein Streitgespräch, Deutsche Richterzeitung 1983, S. 351 ff.

12 Vgl. zuletzt van Bühren, Zeitschrift für Schadensrecht 1993, S. 145.

13 Blankenburg/Fiedler, Die Rechtsschutzversicherungen und der steigende Geschäftsanfall der Gerichte, Tübingen 1981.

14 Luhmann, Rechtssoziologie, 3. Aufl. 1987, S. 190 ff.

15 A.a.O S. $222 \mathrm{ff}$.

16 A.a.O S. 251 ff; ders., Legitimation durch Verfahren, 1969, 2. Aufl. 1975. 
17 Die soziologische Beobachtung des Rechts, 1986, S. 26.

18 Die soziologische Beobachtung des Rechts, S. 27.

19 Gustav Radbruch, Gesetzliches Unrecht und übergesetzliches Recht, 1946; wieder abgedruckt in: ders: Rechtsphilosophie, 8. Aufl. 1973, S. 345.

20 Habermas, Faktizität und Geltung, Frankfurt 1992.

21 A.a.O. S. $37 \mathrm{ff}$.

22 A.a.O. S. 50.

23 A.a.O. S. 50.

24 A.a.O. S. $32 \mathrm{ff}$.

25 A.a.O. S. 19.

26 A.a.O. S. 140, $196 \mathrm{ff}$.

27 A.a.O. S. 46.

28 A.a.O. S. 49.

29 A.a.O. S. 139.

30 A.a.O. S. $137 \mathrm{ff}$.

31 A.a.O. S. 150.

32 A.a.O. S. 225.

33 Vgl. die Besprechungen von Habermas' Buch von Lüderssen, JZ 1993, S. 458; Simon, FAZ vom 8.12.1992; auch Teubner, Frankfurter Rundschau vom 11.11.1992.

34 Vgl. statt aller Böckenförde, Zur Lage der Grundrechtsdogmatik nach 40 Jahren Grundgesetz, Veröffentlichungen der C. F. v. Siemens-Stiftung, München 1990.

35 In der Rechtstheorie ist hier zuletzt auf Alexy, Begriff und Geltung des Rechts, Freiburg/München 1992, zu verweisen. Vgl. ferner die Wiederbelebung der Lehre von Gesellschaftsvertrag durch J. Rawls, A Theory of Justice, 1971, dt.: Eine Theorie der Gerechtigkeit, 1979, und dort vor allem den zweiten Grundsatz der Gerechtigkeit, S. 81 ff. 


\section{Thomas Raiser}

Geboren 1935 in Stuttgart.

Studium zunächst der Philosophie und klassischen Philologie, sodann der Rechtswissenschaft in Tübingen, Bonn, Berlin und München.

1962 Promotion in Tübingen.

1964 - 1965 Rechtsanwalt in Stuttgart.

1969 Habilitation in Hamburg.

1970 - 1992 Ordentlicher Professor an der Universität Gießen.

1977 - 1992 Richter am Kartellsenat des OLG Frankfurt.

1978 Hauptprozeßbevollmächtigter des Deutschen Bundestages im Mitbestimmungsprozeß vor dem Bundesverfassungsgericht. Gastprofessuren an der Universität Madison/Wisconsin, USA und an der Kaiserlichen Universität Tokio.

Wichtigste Veröffentlichungen

Das Unternehmen als Organisation, Kritik und Erneuerung der juristischen Unternehmenslehre, 1969.

Marktwirtschaft und paritätische Mitbestimmung, 1973.

Grundgesetz und paritätische Mitbestimmung, 1975.

Die Aussperrung nach dem Grundgesetz, 1975.

Mitbestimmungsgesetz, Kommentar, 2. Auf., 1984.

Rechtssoziologie, 1987.

Recht der Kapitalgesellschaften, 2. Aufl., 1992.

Gesetz zur Regelung kollektiver Arbeitskonflikte, 1988 (zusammen mit Birk, Konzen, Löwisch und Seiter).

Rechtsschutzversicherungen und Rechtsverfolgung, 1933

(zusammen mit Jagodzinski und Riehl). 
In der Reihe Öffentliche Vorlesungen sind erschienen:

$1 \quad$ Volker Gerhardt

Zur philosophischen Tradition der HumboldtUniversität

2 Hasso Hofmann

Die versprochene Menschenwürde

3 Heinrich August Winkler Von Hitler zu Weimar

Die Arbeiterbewegung und das Scheitern der ersten deutschen Demokratie

$4 \quad$ Michael Borgolte

„Totale Geschichte“ des Mittelalters?

Das Beispiel der Stiftungen

$5 \quad$ Wilfried Nippel

Max Weber und die Althistorie seiner Zeit

$6 \quad$ Heinz Schilling

Am Anfang waren Luther, Loyola und Calvin ein religionssoziologisch-entwicklungs-

geschichtlicher Vergleich

$7 \quad$ Hartmut Harnisch

Adel und Großgrundbesitz im ostelbischen

Preußen 1800 - 1914 


\section{Fritz Jost}

Selbststeuerung des Justizsystems durch richterliche Ordnungen

9 Erwin J. Haeberle

Berlin und die internationale Sexual-

wissenschaft

10 Herbert Schnädelbach

Hegels Lehre von der Wahrheit

11 Felix Herzog

Über die Grenzen der Wirksamkeit des Strafrechts

12 Hans-Peter Müller

Soziale Differenzierung und Individualität.

Georg Simmels Gesellschafts- und Zeitdiagnose

Es erscheinen demnächst:

14 Ludolf Herbst

Der Marshallplan als Herrschaftsinstrument?

Überlegungen zur Struktur amerikanischer Nachkriegspolitik

15 Gert-Joachim Glaeßner

Demokratie nach dem Ende des

Kommunismus 


\section{Arndt Sorge}

Arbeit, Organisation und Arbeitsbeziehungen in Ostdeutschland

\section{Achim Leube}

Semnonen, Burgunden, Alamannen.

Archäologische Beiträge zur germanischen Frühgeschichte 